\title{
STAT3 is involved in IL-6-Mediated Downregulation of Hepatic Transporters in Mice
}

Walaa A. Abualsunun and Micheline Piquette-Miller

Leslie Dan Faculty of Pharmacy, University of Toronto, Toronto, Ontario, Canada m.piquette.miller@utoronto.ca.

Received, October 31, 2018; Accepted, November 20, 2018; Published, November 21, 2018.

\begin{abstract}
Interleukin (IL)-6 decreases hepatic expression of numerous transporters. Although IL-6 signaling occurs through STAT3, the extent of the involvement of the STAT3 signaling pathway has not been elucidated. PURPOSE: Our objective was to investigate whether IL-6-mediated effects occur through STAT3, and whether PXR plays a role in this regulation. METHOD: PXR null (-/-) or wild-type (+/+) male mice were pre-dosed with a selective STAT3 inhibitor S3I-201 (7.5 mg/ $/ \mathrm{kg}$ ip) or vehicle ( $n=5-8 /$ group) 30 minutes before receiving a single dose of IL-6 (1 $\mu \mathrm{g}$ ip) or saline. Animals were sacrificed after 6 hours and liver samples were analyzed using qRTPCR and western blotting. RESULTS: As compared to saline controls, IL-6 decreased the expression of Cyp3a, Abcb1a, Abcc3, and Slco1a4 20-70\% similarly in PXR (+/+) and (-/-) mice at $6 \mathrm{hr}$, while downregulation of Abcb11, Abcc2, Slc10a1 and Slco2b1 was only seen in PXR (+/+). Pre-administration of S3I-201 attenuated IL6-mediated changes of most transporters in PXR (+/+) and PXR (-/-) mice. At early times after IL-6 administration (10-120 minutes), transcript levels of Socs3, PXR, Abcb1a, Abcc3, Abcb11, Slco1a4 and Slco2b1were increased in PXR (+/+) mice. CONCLUSIONS: Our findings demonstrate that IL-6 imposes a significant downregulation of numerous ABC and SLC transporters in liver primarily through activation of the STAT3 signaling pathway. Based on time-dependent changes in transporter expression, downregulation likely occurs downstream of STAT3 activation. As IL-6 is elevated in many diseases, understanding the underlying mechanism(s) involved in transporter dysregulation will allow us to predict potential drug-disease interactions.
\end{abstract}

\section{INTRODUCTION}

IL-6 is one of the key cytokines involved in the inflammatory response and a major regulator of the acute-phase immune response in the liver $[1,2]$. Much evidence has demonstrated that IL-6 significantly downregulates the expression and activity of drug metabolizing enzymes [3-9]. Likewise, a substantial downregulation of numerous ABC and SLC transporters is seen in IL- 6 treated rodents and primary cultured human hepatocytes, demonstrating the importance of this cytokine in the regulation of these drug disposition proteins in the liver [10-13]. However, the underlying mechanism of IL-6-mediated downregulation of hepatic transporters is not fully known.

Activation of pregnane-X-receptor (PXR) is involved in the upregulation of many drug transporters and drug metabolizing enzymes and is also thought to be involved in inflammationmediated downregulation as IL-6 has been shown to decrease PXR expression. Indeed, IL-6-mediated suppression of Abcb11 (Bsep), Abcc2 (Mrp2), and Slcola4 (Oatpla4) was detected in PXR $(+/+)$ mice but not in PXR (-/-) mice, suggesting partial involvement of PXR [14, 15]. IL-6-mediated downregulation of CYP3A4 in human hepatocytes has been reported to occur through PXR downregulation [16]. Moreover, IL-6 mediated downregulation in the expression of PXR and its target genes (P-glycoprotein and CYP3A4 or Cyp3a11) has been demonstrated in primary cultures of both human and mouse hepatocytes, indicating conservation of this regulatory pathway [17]. However, in vivo studies examining direct involvement of PXR following IL-6 administration are lacking.

IL-6 is classically known to activate gene expression via the Signal Transducer and Activator of Transcription (STAT3) signalling pathway [18, 19]. Binding of IL-6 to its receptor IL-6R triggers the tyrosine-kinase activity of the Janus-kinase-family member JAK3.

Corresponding Author: Micheline Piquette-Miller, PhD. Professor, Leslie Dan Faculty of Pharmacy, University of Toronto, 144 College Street, Toronto, ON, E-mail: m.piquette.miller@utoronto.ca 
Activated JAK3 then phosphorylates STAT3 resulting in its dimerization and translocation to the nucleus where it activates transcription of several target genes including other inflammatory cytokines [20, 21] (Fig.1). Indeed, STAT3 plays an important role in cytokine-mediated induction of acute-phase response genes in vivo. Of note, expression of the IL-6/STAT3 target gene, Suppressor of cytokine signaling 3 (Socs 3 ) increases rapidly in response to IL-6; providing a negative feedback loop for regulation of cytokines [22-24].

As the underlying mechanisms responsible for transporter dysregulation following IL-6 administration have not been fully elucidated, our objective was to clarify whether these changes occur through STAT3, and whether PXR is involved. Therefore, we administered a specific inhibitor of STAT3, S3I-201, to PXR (+/+) and PXR (-/-) mice prior to IL-6 administration in order to elucidate the effects on hepatic transporters. S3I-201 selectively binds to the Stat3-SH2 domain thereby blocking Stat3 phosphorylation, STAT3 dimerization and STAT3-dependent transcription. Selective in vivo inhibition of STAT3 activation has been previously demonstrated in rodents with more than 3-fold selectivity for STAT3 over STAT1 [24]. Moreover, Johnson et al. [27] reported that S3I-201 did not prevent lipopolysaccharide (LPS)-induced endothelial dysfunction; effects which are primarily mediated through the NF- $\mathrm{BB}$ signaling pathway. Oral and intraperitoneal doses of $5-10 \mathrm{mg} / \mathrm{kg}$ have been demonstrated to effectively suppress STAT3 activation in vivo in mice $[25,26]$. In order to provide insight into direct or indirect involvement of STAT3 transcriptional activity, the effect of IL-6 at early time points in wild type mice is also evaluated in this study.

\section{MATERIALS AND METHODS}

\section{Animals and Experimental Design}

The animal experiments were performed in accordance to the guidelines of the University of Toronto Animal Care Committee and the Canadian Council on Animal Care. PXR wild-type (+/+) C57BL/6 mice were purchased from Charles River Canada (Montreal, PQ, Canada) and PXR knockout (-/-) C57BL/6 mice were obtained from an ongoing inbred colony [27]. Ten to twelve week-old male PXR (+/+) wild-type and PXR (-/-) knockout mice were treated with a $7.5 \mathrm{mg} / \mathrm{kg}$ intraperitoneal (ip) injection of the selective STAT3 inhibitor S3I-201
(AdooQ Bioscience, Irivine, CA) or vehicle (dimethylsulfoxide/saline). A single dose of IL-6 (R\&D system, Minneapolis, MN) (1 $\mu \mathrm{g}$ ip) or saline was given thirty minutes after administration of S3I201 or vehicle. Downregulation of numerous hepatic transporters has been previously reported at 6 hours following IL-6 administration [10, 14]. Therefore mice ( $n=5-8$ /group) were euthanized six hours after IL-6 or saline administration. In order to examine early transcription events, another set of wild-type C57BL/ 6 mice ( $n=5-6 /$ group) were treated with a single dose of IL-6 (1 $\mu \mathrm{g}$ ip) and sacrificed at early time points $(10,30,60$ and 120 minutes) and compared to vehicle treated controls. Animals were sacrificed at each time point; serum and livers were collected and immediately snap frozen in liquid nitrogen to be stored at $-80^{\circ} \mathrm{C}$ for later analysis.

\section{Quantitative Real-time Polymerase Chain Reaction}

The mRNA levels of each transporter were quantified using qRT-PCR. Methods for RNA isolation, cDNA synthesis and qRT-PCR have been previously described [27]. Briefly, RNA was extracted from liver tissue using TRIzol (Invitrogen, Carlsbad, CA) according to the manufacturer's instruction and reverse transcribed (RT) to single stranded complementary DNA (cDNA) using a High Capacity cDNA RT kit (Applied Biosystems, Foster City, CA). Quantitative Real-time PCR was performed using Power SYBR Green detection system (ABI HT 7900, Applied Biosystems, Streetsville, ON, Canada). The relative mRNA expression was calculated using the comparative $\mathrm{Ct}$ $(\Delta \Delta \mathrm{Ct})$ method and each gene of interest was normalized to the house keeping gene 36B4. Specific primer sequence have been previously published [27].

\section{Western Blotting}

Nuclear protein was extracted from liver tissues as previously described [27]. Briefly, $100 \mathrm{mg}$ of liver tissue was homogenized using homogenizing buffer (250 $\mathrm{mM}$ sucrose, $10 \mathrm{mM}$ HEPES, and $10 \mathrm{mM}$ Tris) containing protease inhibitor cocktail (SigmaAldrich) and centrifuged (12,000 rpm, 15 minutes at $4^{\circ} \mathrm{C}$ ). Then, nuclear pellet was re-suspended in 150 $200 \mu$ nuclear extraction buffer, incubated (30 minutes) and sonicated ( 3 minutes) before the final centrifugation $\left(14,000 \mathrm{rpm}, 10\right.$ minutes at $\left.4^{0} \mathrm{C}\right)$ after which the supernatant was collected. This nuclear protein concentration was quantified using the 
Bradford assay with BSA standards. Forty $\mu \mathrm{g}$ of protein in Laemmli buffer (Biorad, Hercules, CA, USA) was separated using a 10\% SDS PAGE gel and transferred to polyvinylidene difluoride (PVDF) membranes (Bio-Rad Laboratories Canada, Mississauga, ON). Membranes were blocked with $5 \% \mathrm{w} / \mathrm{v}$ skim milk powder in TBST and incubated over night with primary antibodies anti-PXR.1 (A20) 1: 200 and p-Stat3 (sc-8059) 1:200 (Santa Cruz Biotechnology) in 2\% skim milk in TBST. After a series of washes with TBST, membranes were incubated with secondary antibodies (anti-goat 1:3000, anti-mouse 1:5000; Jackson ImmunoResearch Laboratories, Inc., West Grove, PA). Immunodetectable proteins were quantified using Alpha Ease FC imaging software (Alpha Innotech, Santa Clara, CA). $\beta$-actin (AC-15, 1:50000, Sigma-Aldrich) and a calibrator were used to account for loading and gel-to-gel variability.

\section{Transcription Factor DNA-binding (TransAM)} Assays

Forty ng of liver nuclear extract were used to assay the specific transcription factor DNA binding activity using the STAT3 TransAM kit (Active Motif, CA, USA) in accordance with the manufacturer's instructions. Absorbance $(450 \mathrm{~nm})$ with a reference wavelength of $655 \mathrm{~nm}$ was detected using a plate reader.

\section{STATISTICS}

All results were expressed as mean \pm S.E.M. Statistical analysis was performed using the GraphPad Prism 7 software. Two-Way ANOVA analysis, followed by Tukey's test, was used to determine the differences between STAT3 inhibitor and IL-6-treatment groups ( $\mathrm{n}=5-8$ mice per treatment group). The criterion of significance was $(\mathrm{p}<0.05)$.

\section{RESULTS}

\section{Effect of STAT3 Inhibition on IL-6 and Socs3} Expression

Following IL-6 treatment, mRNA expression of the IL-6 target gene Socs3 was significantly increased to a similar extent in both PXR $(+/+)$ and PXR (-/-) (Figure 2 A, B). Prior treatment with the STAT3 inhibitor (S3I-201) significantly suppressed IL-6 and Socs3 induction in both PXR (+/+) and PXR (-/-) mice $(\mathrm{p}<0.05)$.
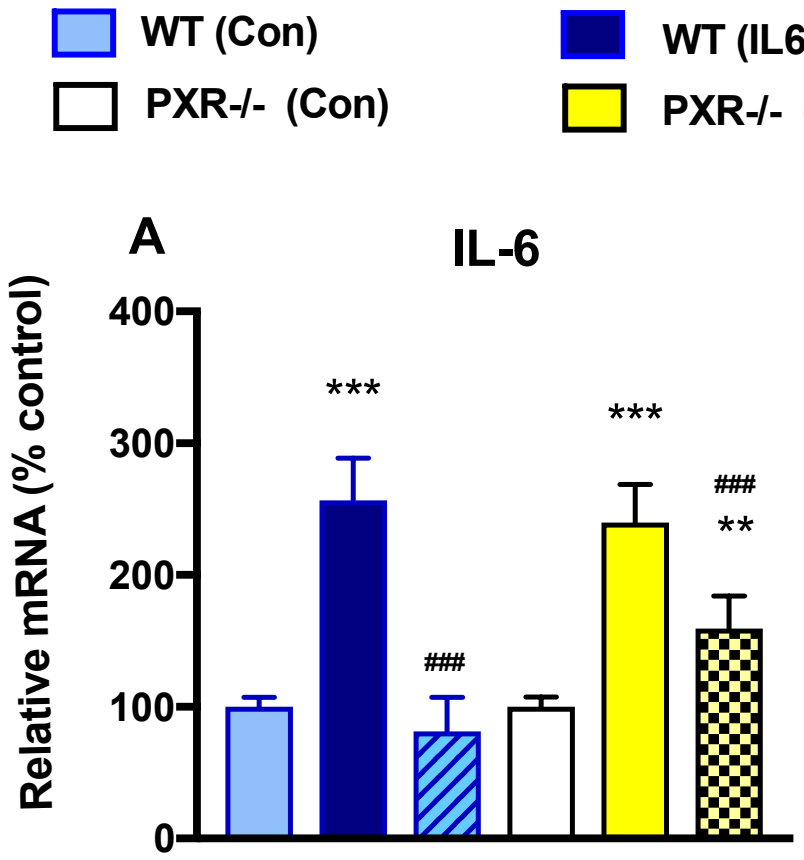
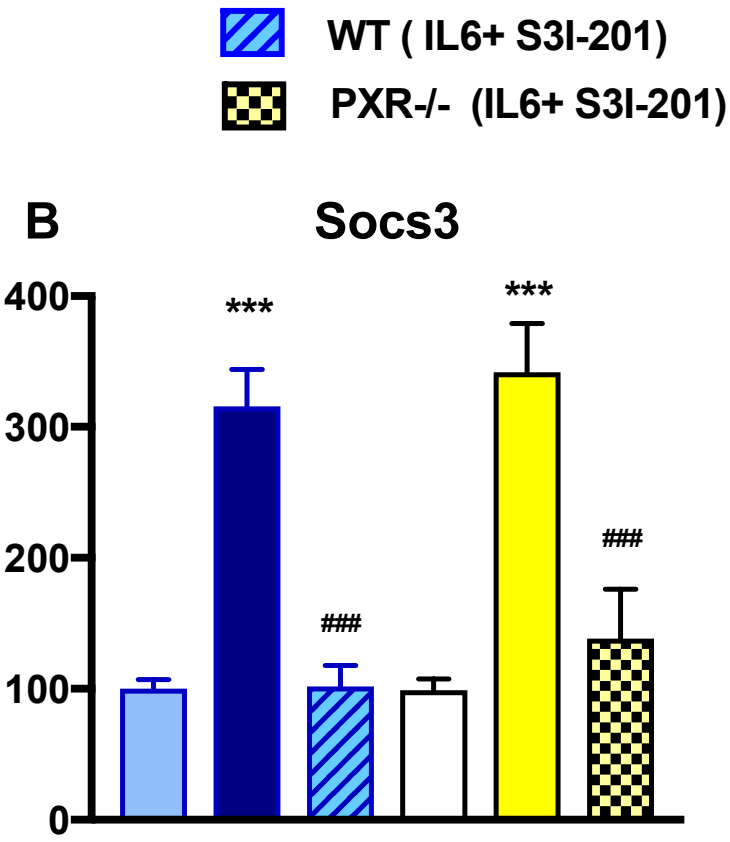

Figure. 2. Effect of S3I-201 on the mRNA levels of (A) IL-6 and (B) IL-6 target gene Socs3 following IL-6 administration in the liver of PXR (+/+) and PXR (-/-) mice. Data are presented as percentage of mean \pm S.E.M $(n=5-8){ }^{*}$ Significant from saline controls and ${ }^{\#}$ significant from IL-6 treated; ${ }^{* *}$,\# $P<0.01$; ${ }^{* * *}, \# \# \# P<0.001$ 


\section{Effect of STAT3 Inhibition on Hepatic Gene Expression}

IL-6 imposed a significant $20-70 \%$ downregulation in the mRNA expression of Cyp3a, Abcb1a, Abcg2, Abcc3, Slc10a1, and Slcola4 in both PXR $(+/+)$ and PXR (-/-) 6 hours after administration (Figure 3). IL6-mediated downregulation of Abcc2, Abcb11, Slc10a1 and Slco2b1 was seen in PXR $(+/+)$ but not PXR (-/-) mice. In PXR (+/+) mice, the mRNA level of PXR was significantly decreased by $50 \%$ following IL-6 treatment relative to control (data not shown). Additionally, pretreatment of PXR $(+/+)$ mice with the STAT3 inhibitor, S3I-201 attenuated IL-6 mediated changes of most transporters compared to controls with the exception of Slcola4. Likewise, attenuation of IL-6 mediated changes of most transporters (Abcb1a, Abcc3, and Slc10a1) was seen in S3I-201 pre-treated PXR (-/-) mice but this did not reach significance for Slcola4 and Abcg2. No attenuation in the downregulation of Cyp3a11 was seen in either PXR (+/+) or (-/-) mice.

\section{Earlier Time Course Treatments with IL-6}

As measured using TransAM assays, binding activity of STAT3 to target DNA was increased 10 minute after IL- 6 treatment, indicating activation of STAT3 signalling (Fig 4A). A significant IL-6mediated increase in the immunodetectable expression of STAT3 was also seen within the nuclear protein fraction at 10 minutes time point (supplementary figure 1). IL-6 also imposed a significant induction in the mRNA expression levels of the IL-6/STAT3 target gene, Socs3 at early time points (Fig 4B).

The early effects of IL-6 on hepatic transporter expression were further examined in PXR $(+/+)$ mice. At early time points, (10-120 minutes), the hepatic expression of Abcb1a, Abcb11 and Slco2b1 were significantly increased and by 60 minutes, levels of Abcc2 and Slcola4 were also increased (Fig 5). Expression of Abcg2, Abcc3, Slco10a1 and Cyp3a11 were not significantly affected at these early time points. Interestingly, a 2-3-fold increase in mRNA and nuclear protein expression of PXR was seen at 30 to 120 minutes after IL-6 administration (Fig 6A,B).

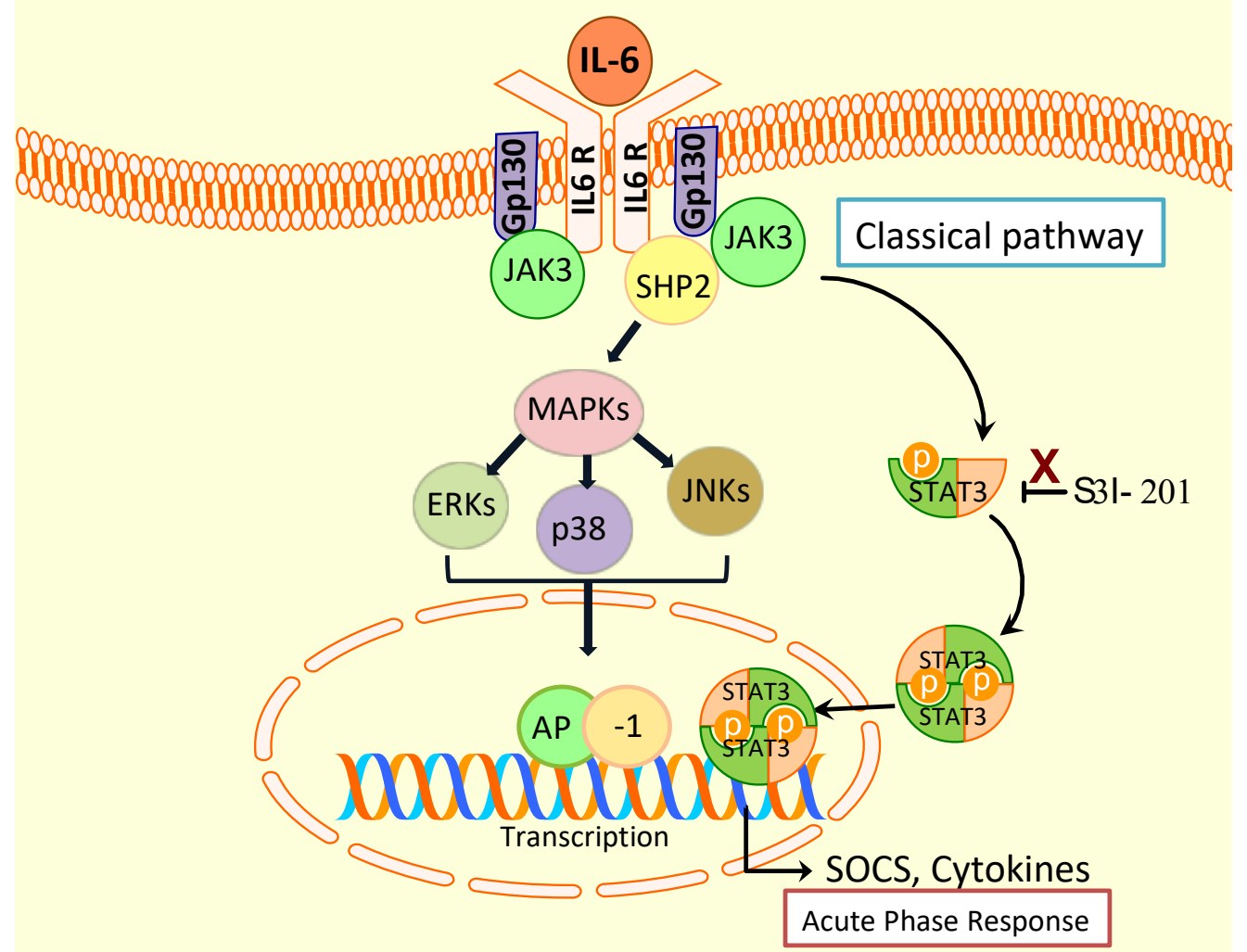

Figure. 1. IL-6 signaling pathway. IL-6 activates gene expression by phosphorylating the Signal Transducer and Activator of Transcription (STAT3), which translocates into the nucleus and activates transcription of acute phase response genes. S3I-201 is highly selective novel inhibitor of STAT3 complex formation that binds to the SH2 domain, inhibits STAT3 phosphorylation and suppresses STAT3-dependent transcriptional activities. 

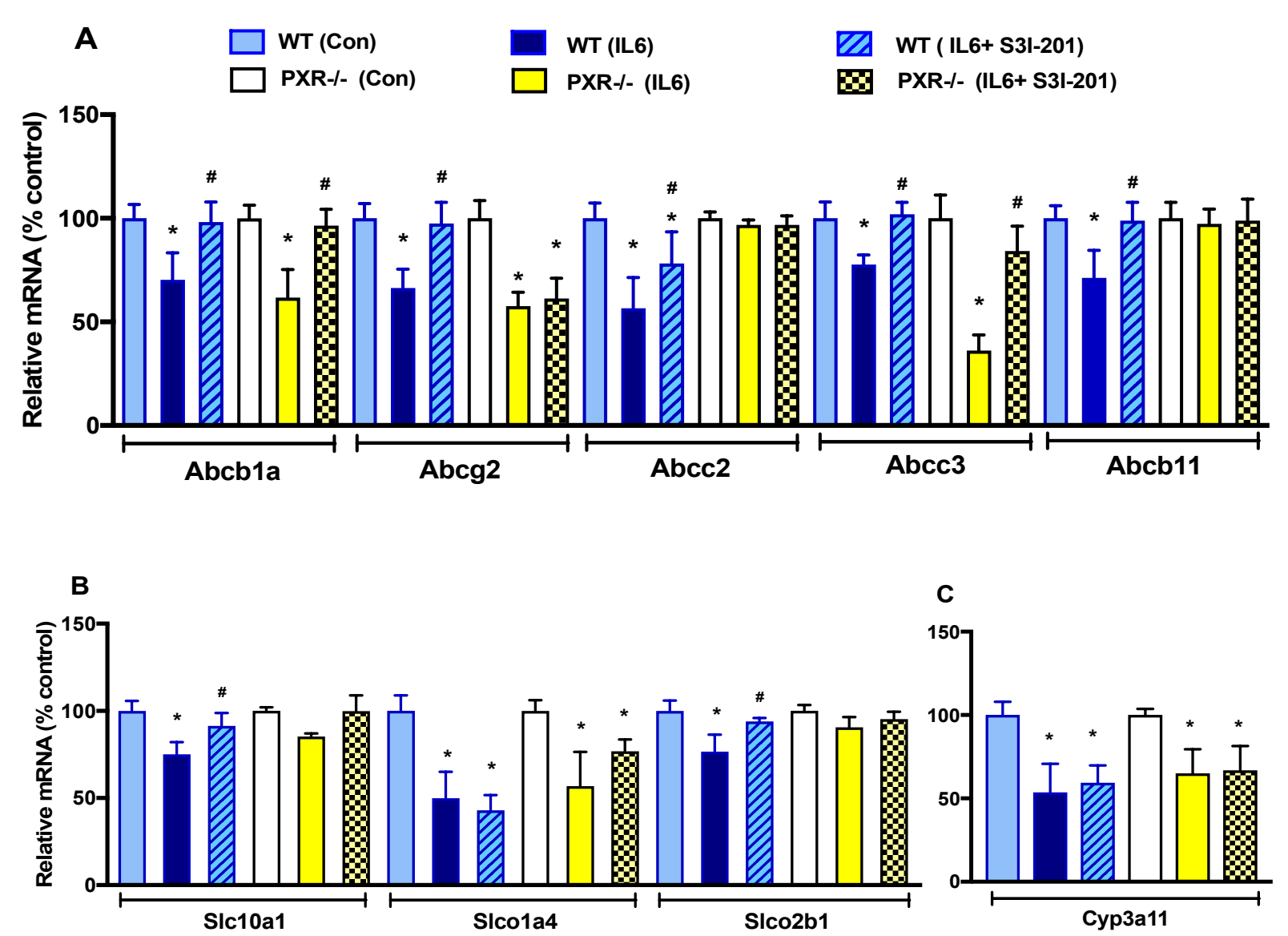

Figure. 3. Effect of S3I-201 pre-treatment on hepatic mRNA expression of (A) ABC transporters, (B) SLC transporters, and (C) Cyp3a11 following IL-6 treatment in PXR (+/+) and PXR (-/-) mice. Controls received saline. Data are presented as percentage of mean \pm S.E.M $(n=5-8) .{ }^{*}$ significant from saline controls and ${ }^{\#}$ significant from IL-6 treated; ${ }^{*},{ }^{*} P<0.05$.
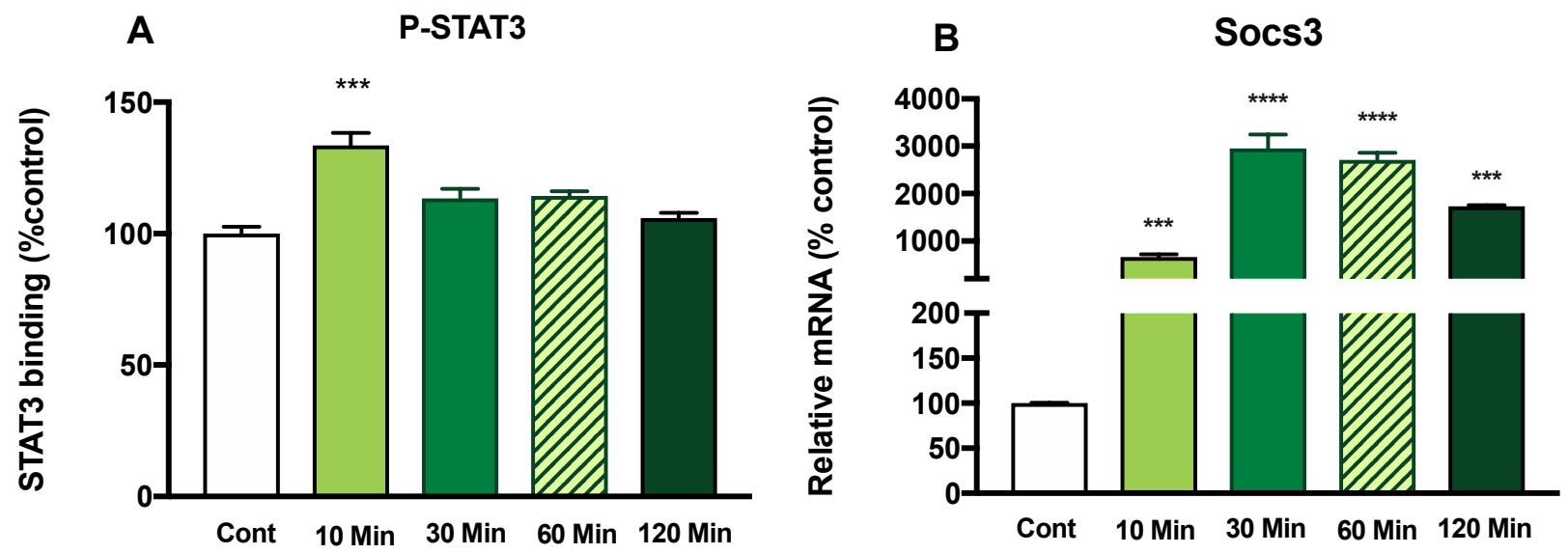

Figure. 4. Effect of IL-6 treatment at early different time points on (A) the hepatic nuclear extract of P-STAT3 as measured by TransAM assay, and (B) the hepatic mRNA expression of Socs3 as measured by qRT-PCR in wild-type mice. Data are presented as percentage of mean \pm S.E.M $(n=5-8) .{ }^{*}$ significant from controls; ${ }^{* *} P<0.01 ;{ }^{* * *} P<0.001$. 

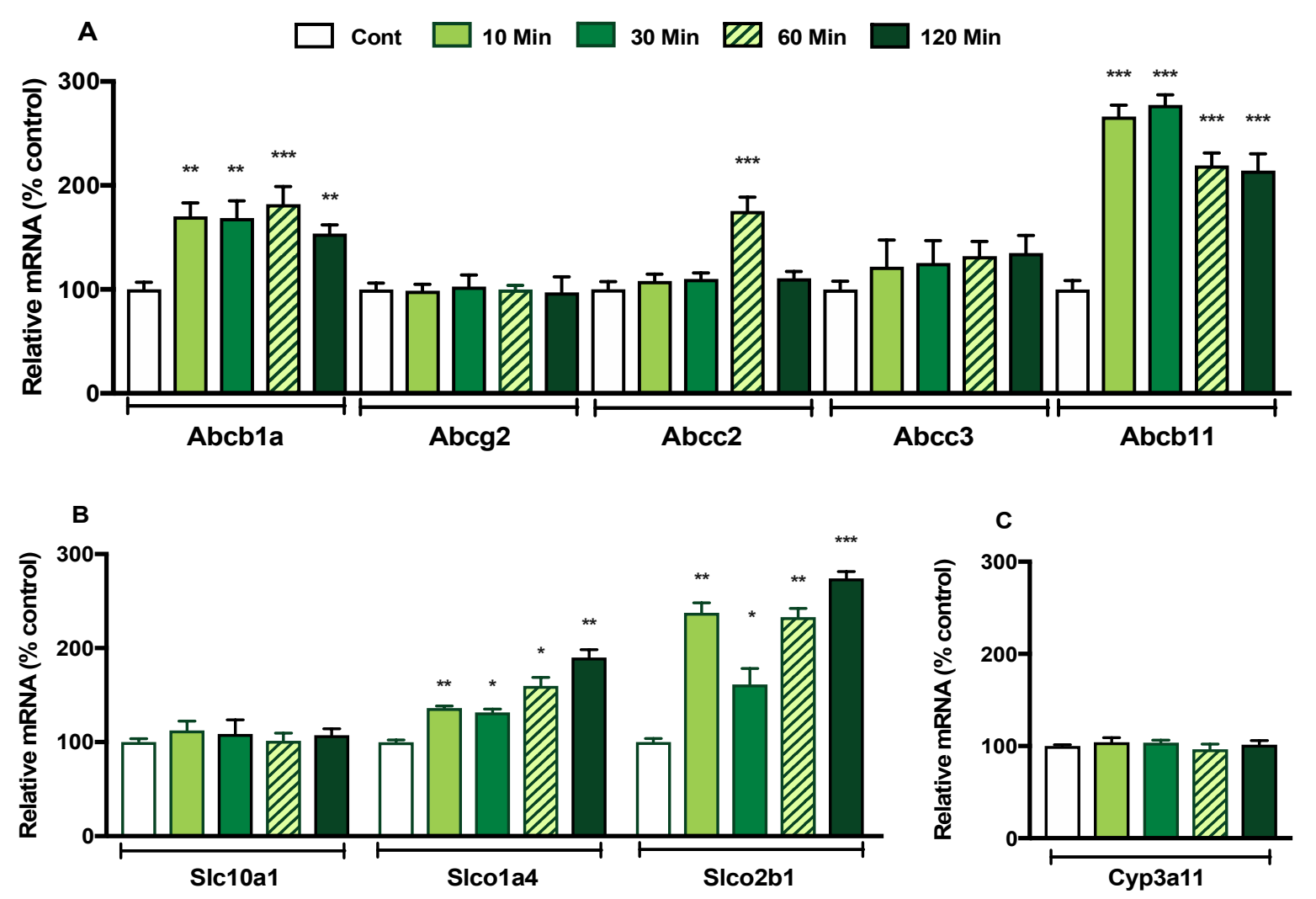

Figure. 5. Effect of IL-6 treatment at early different time points on the hepatic mRNA expression of (A) ABC transporters, (B) SLC transporters, and (C) Cyp3a11 in wild-type mice. Controls received saline. Data are presented as percentage of mean \pm S.E.M $(n=5-8) .{ }^{*}$ significant from controls; ${ }^{*} P<0.05 ;{ }^{* *} P<0.01 ;{ }^{* * *} P<0.001$.
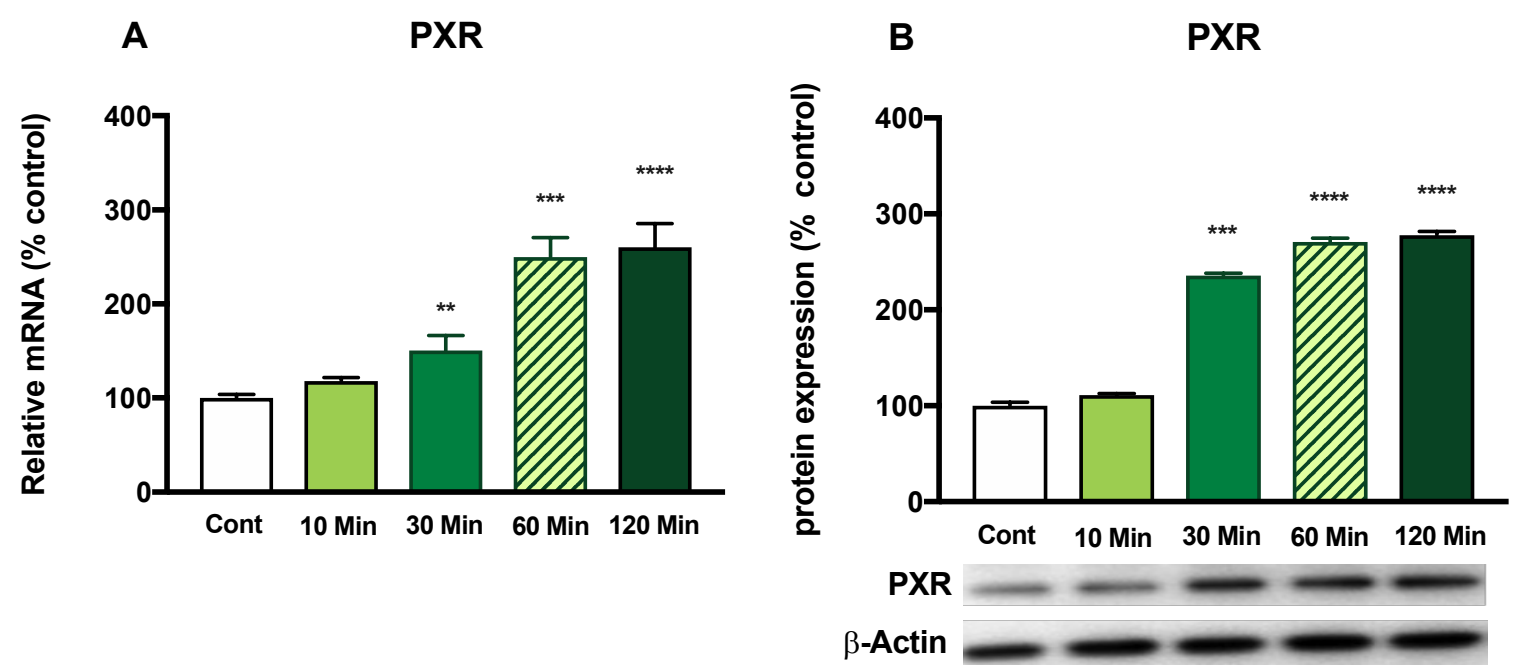

Figure. 6. Effect of IL-6 treatment at early different time points on (A) hepatic mRNA expression of PXR and (B) hepatic nuclear protein expression and representative western blot of PXR in wild-type mice. Controls received saline. mRNA and protein expression were determined as described in Material and Methods. Data are presented as percentage of mean \pm S.E.M $(n=5-8) .{ }^{*}$ significant from controls; ${ }^{* *} P<0.01 ;{ }^{* * *} P<0.001$. 


\section{DISCUSSION}

During inflammation, secretion of IL-6 regulates the expression of numerous genes through activation of the JAK3/STAT3 signalling pathway [18]. Following IL-6 stimulation, dimerization of JAK3 causes phosphorylation and translocation of STAT3 into the nucleus whereby it regulates transcription of numerous genes. STAT3 activation after IL-6 exposure has been reported in cultured human as well as rodent primary hepatocytes [28]. Several in vivo studies in animal models have demonstrated IL6 mediated downregulation of hepatic transporters, nuclear receptors and drug metabolizing enzymes $[15,29]$. Moreover, IL-6- mediated repression of hepatic transporters and metabolizing enzymes have been reported in vitro in primary cultured human hepatocytes and elevated levels of IL-6 are thought to play an important role in clinical disease-drug interactions [30]. However, studies examining the direct involvement of the STAT3 signalling pathway on IL-6 mediated changes in these genes are lacking. Results from our current study demonstrate that IL-6 administration imposes a downregulation of hepatic transporters and Cyp3a11. Our findings also demonstrate that prior administration of the selective STAT3 inhibitor (S3I-201), which was found to fully suppress induction of the IL-6/STAT3 target gene Soc3, attenuated the downregulation of virtually all transporters. These findings reveal involvement of the STAT3 signaling pathway in the regulation of hepatic transporters but not Cyp3a11.

In general, our results indicate that STAT3 inhibition occurs largely independent of PXR but IL6 mediated effects appear to be influenced by PXR. We observed Abcc2, Abcb11, Slc10a1 and Slco2b1 downregulation in IL-6 treated PXR wild-type but not PXR knockout mice, which is consistent with previous reports [14]. This could stem from either strain differences in inflammatory responses to IL-6 or a PXR-dependency on the regulation of these genes. As we found that IL-6 administration significantly increased mRNA levels of the IL-6 target gene Socs 3 to a similar extent in PXR (+/+) and PXR (-/-) mice, this suggests similar responses to IL-6. Moreover, nuclear protein expression of PSTAT3 was not significantly different between IL-6treated PXR (+/+) and (-/-) (data not shown). These findings imply that PXR may play a role in IL-6 mediated downregulation of Abcc2, Abcb11, Slc10a1 and Slco2b1 at least in part. It could also stem from increased dependency on other nuclear factors in the PXR (-/-) mice. However, for the remaining genes, PXR did not appear to play a role in their downregulation.

To examine whether IL-6 directly impacts hepatic gene transcription of transporters through STAT3 binding sites, we examined their expression at early time points in PXR (+/+) mice. As expected, STAT3 was activated within 10 minutes of IL-6 administration along with rapid induction of Socs3. However, rather than downregulation, we found that IL-6 administration caused an induction in the hepatic expression of Abcb1a, Abcb11 and Slco2b1 within 10 minutes and induction of $A b c c 2$ and Slco1a4 within 60 minutes. Time dependent effects of IL-6 have been seen previously in primary cultured human hepatocytes. Consistent with our findings, Yang et al (2012) reported a rapid induction of Soc3 in IL-6 treated hepatocytes along with a downregulation of most ABC and SLC transporters at later time points. Of particular interest, they observed significant increases in the mRNA expression of ABCB11, SLC22A1, ABCB1, $\mathrm{ABCC} 2$ and $\mathrm{ABCG} 2$ after 2-6 hours of IL-6 exposure while downregulation occurred after 24-48 hours. The molecular mechanisms were not examined. To this point we observed a significant 23 -fold induction in the mRNA and nuclear protein expression of PXR at early time points. As many transporters are known to be regulated by PXR it is plausible that rapid PXR activation could be responsible for this early induction. Indeed, it is well established that PXR activation by hormone, bile acids, or xenobiotics lead to the hepatic induction of several murine transporters including Abcb1a, Abcc2, Abcc3, Abcb11, Slco1a4, and Slco2b1 [3134]. However, it is not known whether a period of 10-60 minutes post-treatment is sufficiently long for this induction to occur through PXR-mediated pathways. Moreover, the lack of induction of wellknown PXR target genes such as Cyp3a11 diminishes the credibility of this hypothesis. An alternative explanation for early induction of these particular transporters could involve direct transcriptional activation through STAT3 binding sites within promoter regions. For example, a binding site for STAT3 can be found within the promoter of murine Abcc2. On the other hand, while IL-6 primarily activates STAT3 in hepatocytes, initiation of other signalling pathways such as the mitogen activated protein kinase (MAPK) cascade are also seen and therefore could also contribute to our findings. 
Based on the observed time dependent phenomenon, IL-6-mediated downregulation of hepatic transporters likely occurs through a pathway downstream of STAT3 activation. Although STAT3 activation was seen in the nuclear fraction after IL-6 administration, this induction was transient, suggesting involvement of other transcriptional factors. It is known that STAT3 activation results in significant induction of Socs3 expression, which results in the release of secondary cytokines that are known to activate NF-kB. Our laboratory recently demonstrated involvement of NF- $\mathrm{BB}$ in the downregulation of hepatic transporters after administration of endotoxin [27]. Given these findings, what we see in this study at 6 hours following IL-6 administration is most likely due to STAT3/Socs3-mediated activation of NF- $\mathrm{kB}$. It is important to note that STAT3 is reported to be NF$\kappa \mathrm{B}$ dependently recruited to the STAT3-NF- $\kappa \mathrm{B}$ transcriptional complex that is known to control the transcription of some of the acute phase proteins [35]. Further studies investigating the involvement of NF- $\mathrm{kB}$ following IL-6 administration are warranted.

Overall this study establishes a principal role of the STAT3 signaling pathway in IL-6 mediated downregulation of hepatic transporters with partial involvement of PXR. However, the contribution of $\mathrm{NF}-\kappa \mathrm{B}$ activation requires further investigation. As inflammation-mediated changes in gene expression are thought to be executed through pathways activated by IL- 6 and other pro-inflammatory cytokines, this information can be used to predict changes in the pharmacokinetics and pharmacodynamics of drugs in diseases associated with inflammation.

\section{ACKNOWLEDGMENT:}

Funding for this study was provided by an operating grant from the Canadian Institutes of Health Research [MOP 13346]. W.A is a recipient of the King Abdul-Aziz University Scholarship for Postgraduate Studies. The authors thank Yen Ting Shen for his technical assistance.

\section{REFERENCES}

1. Heinrich PC, Castell JV, Andus T. Interleukin-6 and the acute phase response. Biochem J, 1990. 265: 62136.
2. Castell JV., et al. Interleukin-6 is the major regulator of acute phase protein synthesis in adult human hepatocytes. FEBS Lett., 1989. 242: 237-9.

3. Morgan ET. Regulation of cytochromes P450 during inflammation and infection. Drug Metab Rev, 1997. 29: 1129-88.

4. Morgan ET., et al. Physiological and pathophysiological regulation of cytochrome P450. Drug Metab Dispos, 1998. 26: 1232-40.

5. Renton KW. Cytochrome P450 regulation and drug biotransformation during inflammation and infection. Curr Drug Metab, 2004. 5: 235-43.

6. Renton KW. Regulation of drug metabolism and disposition during inflammation and infection. Expert Opin Drug Metab Toxicol, 2005. 1: 629-40.

7. Aitken AE.,Richardson TA, Morgan ET. Regulation of drug-metabolizing enzymes and transporters in inflammation. Annu Rev Pharmacol Toxicol, 2006. 46: 123-49.

8. Morgan, ET. Impact of infectious and inflammatory disease on cytochrome P450-mediated drug metabolism and pharmacokinetics. Clin Pharmacol Ther, 2009. 85: 434-8.

9. Evers R., et al. Emerging Evidence Indicates that Disease-Associated Changes in Drug Transporters May Impact the Pharmacokinetics and/or Toxicity of Drugs: A White Paper from the International Transporter Consortium. Clin Pharmacol Ther, 2018. 104:900-15.

10. HartmannG., Cheung AK, Piquette-Miller M. Inflammatory cytokines, but not bile acids, regulate expression of murine hepatic anion transporters in endotoxemia. J Pharmacol Exp Ther, 2002. 303: 27381.

11. Hartmann G, Kim H, Piquette-Miller M. Regulation of the hepatic multidrug resistance gene expression by endotoxin and inflammatory cytokines in mice. Int Immunopharmacol, 2001. 1:189-99.

12. Siewert E., et al. Hepatic cytochrome P450 downregulation during aseptic inflammation in the mouse is interleukin 6 dependent. Hepatology, 2000. 32: 4955.

13. Yang Q., et al. Effects of culture duration on gene expression of P450 isoforms, uptake and efflux transporters in primary hepatocytes cultured in the absence and presence of interleukin-6: implications for experimental design for the evaluation of downregulatory effects of biotherapeutics. Curr Drug Metab, 2012. 13: 938-46.

14. Teng S, Piquette-Miller M. The involvement of the pregnane $X$ receptor in hepatic gene regulation during inflammation in mice. J Pharmacol Exp Ther, 2005. 312: 841-8.

15. Morgan, ET., et al. Regulation of drug-metabolizing enzymes and transporters in infection, inflammation, and cancer. Drug Metab Dispos, 2008. 36: 205-16. 
16. Yang J., et al. Pregnane $X$ receptor is required for interleukin-6-mediated down-regulation of cytochrome P450 3 A4 in human hepatocytes. Toxicol Lett, 2010. 197: 219-26.

17. Ning R., et al. Interleukin-6 Induces DEC1, Promotes DEC1 Interaction with RXRalpha and Suppresses the Expression of PXR, CAR and Their Target Genes. Front Pharmacol, 2017. 8: 866.

18. Heinrich PC., et al. Principles of interleukin (IL)-6type cytokine signalling and its regulation. Biochem J, 2003. 374:1-20.

19. Akira S., IL-6-regulated transcription factors. Int J Biochem Cell Biol, 1997. 29: 1401-18.

20. Streetz KL., et al. Interleukin 6 and liver regeneration. Gut, 2000. 47: 309-12.

21. Taub R. Liver regeneration: from myth to mechanism. Nat Rev Mol Cell Biol, 2004. 5: 836-47.

22. Kishimoto T. Interleukin-6: from basic science to medicine--40 years in immunology. Annu Rev Immunol, 2005. 23: 1-21.

23. Babon JJ., et al. Suppression of cytokine signaling by SOCS3: characterization of the mode of inhibition and the basis of its specificity. Immunity, 2012. 36: 239-50.

24. Carow B, Rottenberg ME. SOCS3, a Major Regulator of Infection and Inflammation. Front Immunol, 2014. 5:58.

25. Wang Z., et al. The STAT3 inhibitor S3I-201 suppresses fibrogenesis and angiogenesis in liver fibrosis. Lab Invest, 2018.

26. Du Y., et al. S3I-201 ameliorates tubulointerstitial lesion of the kidneys in MRL/lpr mice. Biochem Biophys Res Commun, 2018. 503:177-180.

27. Abualsunun WA, Piquette-Miller M., Involvement of Nuclear Factor kappaB, not Pregnane X Receptor, in
Inflammation-Mediated Regulation of Hepatic Transporters. Drug Metab Dispos, 2017. 45: 10771083.

28. Gao B., Cytokines, STATs and liver disease. Cell Mol Immunol, 2005. 2: 92-100.

29. Cressman AM, Petrovic V, Piquette-Miller M., Inflammation-mediated changes in drug transporter expression/activity: implications for therapeutic drug response. Expert Rev Clin Pharmacol, 2012. 5: 6989.

30. Evers R., et al. Disease-Associated Changes in Drug Transporters May Impact the Pharmacokinetics and/or Toxicity of Drugs: A White Paper From the International Transporter Consortium. Clin Pharmacol Ther, 2018. 104: 900-915.

31. Teng S, Jekerle V, Piquette-Miller M. Induction of ABCC3 (MRP3) by pregnane X receptor activators. Drug Metab Dispos, 2003. 31: 1296-9.

32. Geick A, Eichelbaum M, Burk O. Nuclear receptor response elements mediate induction of intestinal MDRI by rifampin. J Biol Chem, 2001. 276: 145817.

33. Staudinger JL., et al. The nuclear receptor $P X R$ is a lithocholic acid sensor that protects against liver toxicity. Proc Natl Acad Sci U S A, 2001. 98: 336974.

34. Watanabe A., et al. Aberrant DNA methylation of pregnane $X$ receptor underlies metabolic gene alterations in the diabetic kidney. Am J Physiol Renal Physiol, 2018. 314: F551-F560.

35. Hagihara K., et al. Essential role of STAT3 in cytokine-driven NF-kappaB-mediated serum amyloid A gene expression. Genes Cells, 2005. 10: 1051-63. 


\section{Supplementary data}

Table 1. Gene and common names of ABC and SLC transporters of interest in the Liver

Gene

Common Name

\begin{tabular}{ccc} 
& ATP-Binding Cassette (ABC) Transporters & \\
\hline Abcb1a & Mdr1a \\
Abcg2 & Bcrp \\
Abcc2 & Mrp2 \\
Abce3 & Bsep \\
Abcb11 & Solute Carrier (SLC) Transporters & Ntcp \\
& Oatp1a4 \\
Slc10a1 & Oatp2b1 \\
\hline
\end{tabular}

\section{Supplementary (Figure 1.)}
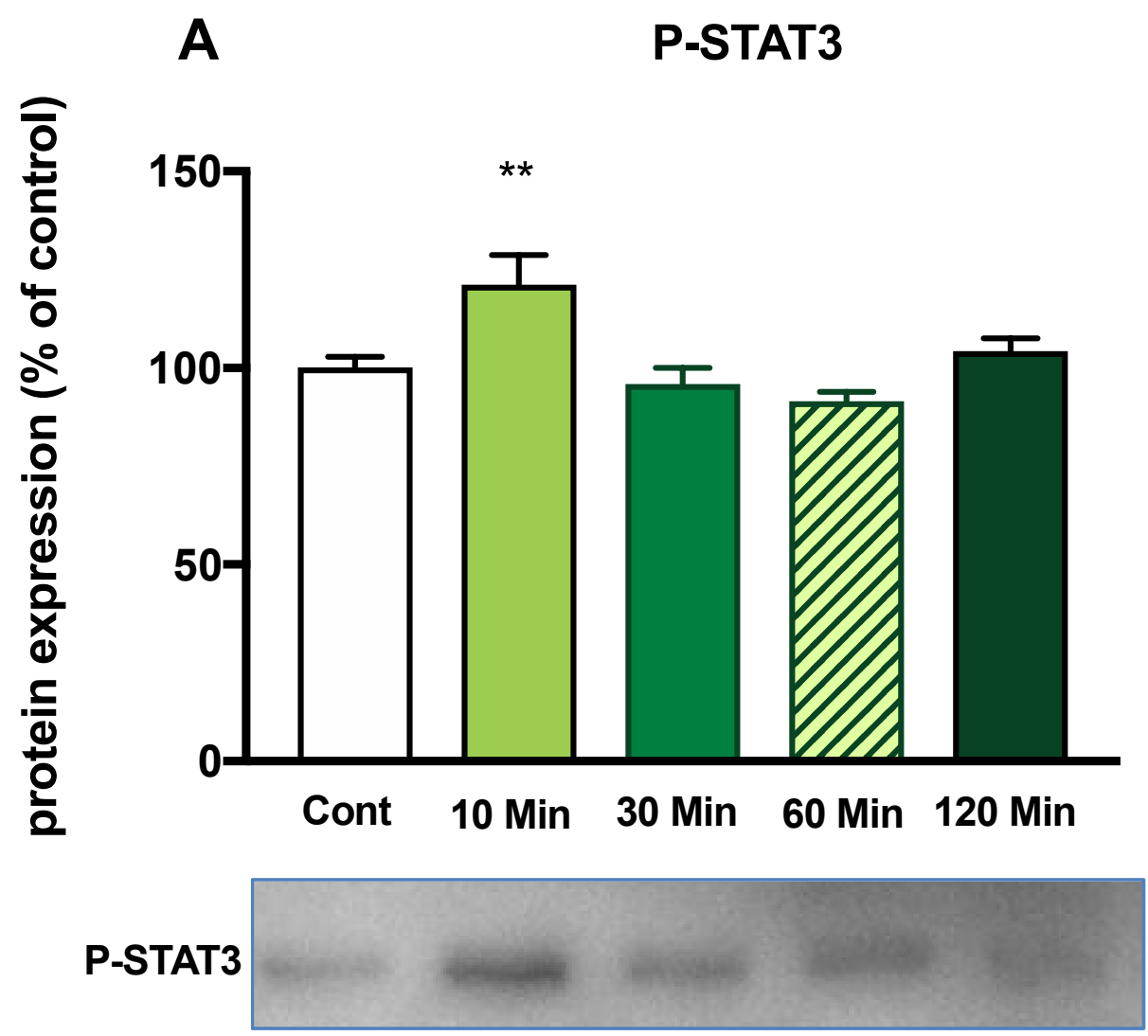

$\beta$-Actin

Supplementary Figure 1. Effect of IL-6 treatment at early different time points on (A) the hepatic nuclear extract of PSTAT3 as measured by Western blot in wild-type mice. Data are presented as percentage of mean \pm S.E.M $(n=5-8)$. significant from controls; ${ }^{* *} P<0.01 ;{ }^{* * *} P<0.001$. 